\title{
Portable Microwave Instrumentation System For Determination Of Moisture Content In Oil Palm Fruits.
}

\begin{abstract}
In this paper, we present an improved instrumentation system for moisture content determination in oil palm fruit. The results obtained using the developed system are compared with the data obtained using the vector network analyzer, values calculated from finite element method (FEM) simulation and capacitance model at $2 \mathrm{GHz}$. This system is found to be more practical, cost effective, and accurate for in situ measurements than existing techniques.
\end{abstract}

Keyword: Instrumentation; Sensor; Microwave. 